\title{
A RELAÇÃO DA ATIVIDADE FÍSICA E A QUALIDADE DE VIDA NA POPULAÇÃ O IDOSA: ANÁLISE DA PRODUÇÃO CIENTIFICA BRASILEIRA
}

\author{
LA RELACIÓN DE LA ACTIVIDAD FÍSICA Y LA CALIDAD DE VIDA EN LA \\ POBLACIÓN DE EDAD AVANZADA: ANÁLISIS DE LA PRODUCCIÓN CIENTÍFICA \\ BRASILEÑA
}

\section{THE RELATIONSHIP OF PHYSICAL ACTIVITY AND THE QUALITY OF LIFE IN THE ELDERLY POPULATION: ANALYSIS OF THE BRAZILIAN SCIENTIFIC PRODUCTION}

\author{
Douglas PRADO ARAÚJO 1 \\ Stela LOPES SOARES ${ }^{2}$ \\ Diogo Queiroz ALLEN PALÁCIO ${ }^{3}$
}

RESUMO: Trata-se de uma revisão integrativa com o objetivo de analisar a produção científica brasileira sobre a influência da atividade física na melhoria da qualidade de vida do idoso. Selecionaram-se artigos publicados em diferentes bases de dados, com descritores: "Exercício" "Qualidade de Vida", "Idoso", "Idoso de 80 anos ou mais". Como critérios: texto completo disponível, em português, limite idoso. O Sudeste tem a maior parte da produção nacional e a maioria delas, tem abordagem quantitativa. Evidenciaram-se ainda a escassa e recente produção científica na temática, o que se contrapõe a ampliação do acesso desta população as práticas corporais e de atividade física.

PALAVRAS-CHAVES: Exercício. Qualidade de vida. Idoso.

RESUMEN: Se trata de una revisión integradora con el objetivo de analizar la producción científica brasileña sobre la influencia de la actividad física en la mejora de la calidad de vida de los ancianos. Se seleccionaron los artículos publicados en diferentes bases de datos, con descriptores: "ejercicio" "calidad de vida", "anciano", "80 años o más". Como criterios: texto completo disponible en inglés, límite antiguo. El sureste tiene la mayor parte de la producción nacional y la mayor parte de ellos tienen acercamiento cuantitativo. Hubo también la escasa y reciente producción científica en la temática, que contrasta la expansión del acceso de esta población a las prácticas corporales y a la actividad física.

1 Universidade Estadual Vale do Acaraú - UVA, Sobral - CE. Especialista em Saúde da Família. Graduado em Educação Física. ORCID: <http://orcid.org/0000-0002-6195-521X>. E-mail: douglaspradomsn@ hotmail.com

2 Centro Universitário INTA (INTA/UNINTA), Sobral - CE - Brasil. Professora do Curso de Educação Física Mestre em Ensino na Saúde, pela Universidade Estadual do Ceará (UECE). Graduada em Educação Física. Especialização em Saúde da Família Membro do Grupo de Estudos e Pesquisa em Educação Física Escolar (GEPEFE). ORCID: <http://orcid.org/00000002-5792-4429>. E-mail: stelalopesoares@ hotmail.com.

3 Secretaria de Educação do Ceará (SEDUC), Fortaleza - CE - Brasil. Professor. Mestre em Ensino na Saúde pela Universidade Estadual do Ceará (UECE). Graduado em Educação Física. Membro do Grupo de Estudos e Pesquisa em Educação Física Escolar (GEPEFE). ORCID: <http://orcid.org/0000-0002-3814-9607>. E-mail: diogoallenpalacio@gmail.com 
PALABRAS CLAVE: Ejercicio. Calidad de vida. Viejo.

ABSTRACT: It is an integrative review with the objective of analyzing the Brazilian scientific production on the influence of physical activity on the quality of life of the elderly. Articles published in different databases were selected, with descriptors: "Exercise", "Quality of Life", "Elderly", "Elderly aged 80 years and over". As criteria: full text available, in English, old age limit. The Southeast has most of the national production and most of them have a quantitative approach. The scarce and recent scientific production on the subject was also evidenced, which contrasts with the expansion of this population's access to physical practices and physical activity.

KEYWORDS: Exercise. Quality of Life. Elderly. Aged 80 years or more.

\section{Introdução}

O aumento da população idosa teve início no final do século XIX nos países mais desenvolvidos, já nos países em desenvolvimento, esse aumento começou a acontecer a partir da metade do século XX (FERREIRA, 2011).

No contexto brasileiro, emergiu nas últimas décadas um novo padrão demográfico que se caracteriza pela redução da taxa de crescimento populacional e por transformações profundas na composição de sua estrutura etária, com um significativo aumento do contingente de idosos (IBGE, 2009).

Entende-se por idoso, no território nacional, as pessoas com idade igual ou superior a 60 anos e esta definição é legalmente instituída pelo Estatuto do Idoso (BRASIL, 2009). De acordo com o IBGE, a população com 60 anos ou mais no Brasil era de 20.590 .599 pessoas em 2010 e em 2012, segundo estimativas, atingiu 20.889.849.

Os grandes centros urbanos, embora já apresentem um perfil demográfico semelhante ao dos países mais desenvolvidos, ainda não dispõem de uma infraestrutura de serviços que dê conta das demandas decorrentes das transformações demográficas vigentes (IBGE, 2009).

Este aumento da população idosa no Brasil gera a necessidade de mudanças nas estruturas sociais para que estas pessoas, ao terem suas vidas prolongadas não fiquem incapacitadas fisicamente e nem sofram com a dependência e consequentemente com a falta de qualidade de vida.

Santos (2004) conceitua qualidade de vida como o total bem-estar pessoal e que abrange uma série de outros aspectos como autoestima, estado emocional, interação social, autocuidado, estado de saúde e valores culturais. 
Independentemente da concepção adotada do instrumento indicador utilizado sobre qualidade de vida, existe uma íntima relação entre este campo de conhecimento, a área da saúde e a prática de atividade física (ALMEIDA; GUTIERREZ; MARQUES, 2012)

Nesta perspectiva, pesquisas apontam que a grande maioria dos órgãos do corpo humano tem a capacidade de funcionar quase tão bem na fase da velhice como na juventude, entre aqueles que praticam estilos de vida saudáveis, como a atividade física diária (ETCHEPARE; PEREIRA; ZINN, 2004)

A promoção de "estilos de vida saudáveis" é encarada pelo Sistema de saúde como uma ação estratégica. Nesse processo, alguns aspectos são facilitadores para a incorporação da prática corporal/ atividade física, como o incentivo de amigos e familiares, a procura por companhia ou ocupação, alguns programas específicos de atividade física e, principalmente, a orientação do profissional de saúde estimulando a população idosa a incorporar um estilo de vida mais saudável e ativo (BRASIL, 2006).

Nos últimos anos o Brasil teve um crescimento considerável em programas de atividade física para a terceira idade, destacando-se as experiências promovidas nas Universidades (BENEDETTI; GONÇALVES; MOTA, 2007).

No entanto, a implantação dos Núcleos de Apoio à Saúde da Família (NASF), criados pelo Ministério da Saúde em 2008, possibilitaram a inserção de diversas categorias profissionais, entre elas o educador físico, na Atenção Básica o que tem ampliado as práticas de atividade física para melhoria da qualidade de vida da população idosa.

Outra estratégia para incremento do nível de atividade física da população brasileira foi a criação do Programa Academia da Saúde que visa contribuir para a promoção da saúde da população a partir da implantação de espaços públicos construídos com infraestrutura, equipamentos e profissionais qualificados para o desenvolvimento de práticas corporais; orientação de atividade física; promoção de ações de segurança alimentar e nutricional e de educação alimentar, bem como outras temáticas que envolvam a realidade local (BRASIL, 2013). Em abril de 2014, segundo dados do Cadastro Nacional de Estabelecimentos de Saúde (CNES), 832 Academias da Saúde estavam implantadas no Brasil, sendo 51,44\% na região Nordeste.

Diante do exposto, torna-se relevante analisar a produção científica sobre influência da atividade física para a melhoria da qualidade de vida nesta população.

\section{Metodologia}


Trata-se de uma revisão integrativa, que se fundamenta em dados publicados e é utilizada para mensurar as características de determinadas temáticas, o que de certa forma indica a sua produção científica (VANTI; 2002).

A revisão integrativa é um método que proporciona a síntese de conhecimento e a incorporação da aplicabilidade de resultados de estudos significativos na prática abordagem quantitativa e estatística para acompanhar o desenvolvimento científico e de padrões de autoria, publicações e o uso dos resultados de investigação (CARVALHO; SILVA; SOUZA, 2010).

A base de dados consultada foi a Biblioteca Virtual em Saúde (BVS), através do site: http://www.bireme.br. A consulta integrou as seguintes bases de dados científicas: Literatura Latino-Americana e do Caribe em Ciências da Saúde (LILACS), IBECS, Literatura Internacional em Ciências da Saúde (MEDLINE), Biblioteca Cochrane de revisões sistemáticas, Scientific Electronic Library Online (SciELO). O estudo foi realizado durante o mês de novembro de 2017.

A busca teve como descritor as palavras "atividade física", no entanto, na definição do DECS significa 'atividade física de um humano ou de um animal como um fenômeno comportamental', o que não se enquadrou com o que a pesquisa se propõe a analisar. No entanto, ao diferenciar 'atividade física' de 'exercício', que significa atividade física geralmente regular e feita com a intenção de melhorar ou manter a aptidão física ou a saúde, o próprio DECS sugeriu o descritor que se adequou à proposta de investigação da pesquisa. Os demais descritores tiveram suas definições aceitas dentro da perspectiva metodológica do trabalho, sendo estes: qualidade de vida, idoso e idoso de 80 anos ou mais.

A pesquisa utilizou os seguintes descritores no campo busca avançada da BVS: 'exercício' and 'qualidade de vida' and 'idoso' or 'idoso de 80 anos ou mais' e encontrou 7.092 publicações que foram filtradas segundo os seguintes critérios em sequência: texto completo disponível na internet (4152), publicações em português (810), limite idoso (2899). Os artigos estavam disponíveis nas seguintes bases de dados: LILACS (36), MEDLINE (740), Sec. Est. Saúde SP (1).

Após a determinação da amostra foi feita uma leitura exploratória e constatou-se que apenas 84 publicações estavam relacionadas a temática, dessas, somente 32 são pesquisas relacionadas à qualidade de vida em idosos através da prática de alguma atividade física. Foram excluídos 52 artigos após a leitura dos resumos, pois abordavam o tema qualidade de vida, mas não relatavam a influência da atividade física para a mesma, bem como os artigos repetidos (publicados em mais de uma base de dados). 


\section{Resultados e discussão}

As publicações sobre a referida temática só aparecem a partir dos anos 2000. Entre 2002, 2004 e 2014 não houve publicações. As publicações concentraram-se nos anos de 2009 a 2011 (seis artigos cada ano), 2001 e 2013 (uma publicação), 2005, 2006, 2008 (duas publicações cada ano), 2007 e 2012 (três publicações), demonstrando que deve ser incentivada à pesquisa em saúde do idoso.

A maioria das publicações está concentrada na região Sudeste, 17 (53\%), seguida das regiões Sul e Nordeste, com cinco publicações (16\%) cada. As regiões Centro-Oeste e Norte possuem, respectivamente, três $(9 \%)$ e duas $(6 \%)$ do total de publicações. Na região Nordeste das cinco publicações, duas foram realizadas no Ceará o que demonstra interesse dos pesquisadores por esta temática no Estado.

Entre os artigos selecionados, notou-se uma abordagem da atividade física, na maioria dos casos, sempre relacionada com a qualidade de vida e/ou alguma patologia. Dentre as 36 publicações destacam-se 16 artigos (37,5\%) que fazem uma relação das atividades físicas, dos exercícios e de atividades terapêuticas com o objetivo da melhoria da qualidade de vida da população pesquisada, seja através de um treinamento resistido, de projetos terapêuticos que envolvam a prática regular de movimentos corporais ou de qualquer outro modo de atividade física.

As demais pesquisas fazem uma associação da qualidade de vida com algum outro fator predominante, tais como: a melhoria das dores em paciente com osteoartrite do joelho, aumento da autoestima, da capacidade funcional e pulmonar, melhora da independência funcional, evolução da relação com pacientes institucionalizados, melhoria do equilíbrio e domínio físico corporal, redução da dor em paciente com osteoporose e em pacientes claudicantes, maior controle do metabolismo, da glicose e de lipídeos e melhor qualidade de vida em paciente aposentados.

No estudo de Imoto; et al (2012), retrata que os aspectos da qualidade de vida que apresentaram uma melhora estatisticamente significante foram: capacidade funcional, aspectos físicos, dor, vitalidade e estado geral de saúde, esta afirmação compactua com o estudo de Pimenta; et al (2008), no qual a prática regular de atividade física apresenta diferença significativa nos domínios "capacidade funcional”, "estado geral de saúde", "vitalidade", "aspectos sociais" e "saúde mental", compactuando assim o que se verificou nos demais estudos, que associaram a atividade física a uma melhora da qualidade de vida. 
Denotando o que foi apresentado no parágrafo anterior sobre os benefícios da atividade física, Lima; et al (2013) em seu estudo avaliou a intensidade da dor pela Escala Visual (protocolo de testes) e durante a mesma observou-se a redução significativa após o programa de exercício, tal fato traz um importante dado que leva à procura de auxílio médico em idosos: as dores. Desta forma, observa-se a atividade física como um importante fator de prevenção de sinais e sintomas de possíveis doenças já instaladas.

Comprovando através de dados, o estudo de Kokubun et al (2007) traz evidências de melhoras no estado de saúde dos idosos, constatados pelos exames clínicos e laboratoriais, tais como redução da pressão arterial, diminuição de lipídios sanguíneos e glicemia, além da redução no número de queixas e de consultas médicas não agendadas nos Postos de Saúde.

A prática regular de exercício físico, a partir do que diz Kannus e Khan (2001) pode melhorar a deambulação, reação de tempo e a força muscular, equilíbrio, coordenação, propriocepção, inclusive de indivíduos com mais de 70 anos.

Toscano e Oliveira (2009) vem mostrar como a atividade física influencia a qualidade de vida entre os grupos de idosas mais ativas em relação às menos ativas. Em seus resultados, os indivíduos mais ativos foram significativamente superiores aos menos.

Para que se consiga tais resultados segundo Pedrinelli et al (2009) precisa que se direcione os exercícios objetivando a melhora das limitações funcionais que o idoso venha a apresentar (dor, amplitude de movimento reduzida ou fraqueza muscular). Além de ser individualizados conforme as necessidades específicas do idoso.

Ainda trazendo dados comprovando a relevância que a atividade física tem na referida população, Ferreira et al (2005) pontua fortes evidências de que um programa de intervenção de 12 semanas enfocando a orientação de atividade física ou a orientação nutricional ou as duas combinadas, promoveu aumento da frequência e duração da prática de atividades físicas moderadas.

Dessa forma, pode-se compreender que a atividade física bem direcionada, orientada por um profissional capacitado, só traz ônus para saúde das pessoas que a buscam, como foi o caso do estudo de Carlos et al (2012), ao evidenciar quando o que foi investigado não trouxe o que o mesmo esperava, procurou justificou que o programa de exercícios físicos influenciou na melhoria global dos pacientes, comprovando tal relevância.

Compactuando com os achados anteriores, o estudo de Ritter e Azevedo (2007) apresenta resultados positivos, mas traz um dado um pouco diferente dos demais, já que no mesmo, o nível de atividade física das idosas obesas não é o fator determinante quando se analisa ações cotidianas simples como o ato de sentar e pegar um objeto do solo. Observou-se, 
ainda, que a simples introdução de algumas informações sobre uma forma biomecanicamente econômica na realização das ações foram suficientes para possibilitar um incremento de uma melhor performance.

Por tal fato, faz-se necessário a conscientização para a busca por um estilo de vida ativo durante o envelhecimento, sensibilizando a população sobre a possibilidade de ser fisicamente ativo sem precisar ter muito tempo e habilidades, conhecimentos ou equipamentos específicos.

No geral, $75 \%$ das publicações são de abordagem quantitativa, isso se dá porque geralmente as pesquisas de campo buscam exprimir as relações dos fenômenos por meio de dados métricos com uma amostra grande. Já as pesquisas com abordagem qualitativa, são revisões de literatura ou pesquisas de campo com amostras pequenas. E a pesquisa quantiqualitativa foi um estudo de corte transversal.

De acordo com Terence e Filho (2006), a pesquisa com abordagem qualitativa, é utilizada para aprofundar-se na compreensão dos fenômenos que estuda, da atuação dos indivíduos, grupos ou organizações em sua ambiência e contexto social, interpretando-os segundo a concepção dos participantes da situação enfocada, sem se preocupar com representatividade numérica. Já a pesquisa quantitativa preocupa-se em medir (qualidade, frequência e intensidade) e analisar as relações causais entre as variáveis.

Os serviços de saúde foram os principais campos de aplicação das pesquisas (40,6\%). A escolha dos profissionais de Educação Física por este campo talvez se deu por estes concentrarem ou possuírem um controle maior da população idosa de cada território. Os estudos de Kokubun et al (2007) contribuem dizendo que além de o programa de atividade física oferecido nas Unidades Básicas de Saúde (UBS) de Rio Claro alcançar uma parcela da população carente de atendimento desta natureza, os exercícios físicos de intensidade baixa a moderada realizados duas vezes por semana nas UBS se mostraram efetivos em melhorar o desempenho de alguns componentes da aptidão funcional e qualidade de vida relacionada à saúde dos participantes. Ainda complementam dizendo que resultados apresentados e a presença das UBS na grande maioria dos municípios brasileiros, se acredita que a implantação de um programa desta natureza seja uma iniciativa viável tanto do ponto de vista social quanto de promoção da saúde.

\section{Considerações finais}


A pesquisa evidenciou escassa e recente produção científica nacional sobre a influência da atividade física para a melhoria da qualidade de vida da população idosa brasileira.

Aponta-se a necessidade de incentivo e fomento à pesquisa para que se ampliem as evidências científicas sobre a temática no Brasil. Igualmente, dada a diversidade nas regiões brasileiras em relação à infraestrutura, renda, educação e outros determinantes sociais, observou-se que há uma necessidade de mais pesquisas nas regiões norte e nordeste para elucidar a influência destes determinantes macrossociais na prática regular de atividade física.

Iniciativas recentes para ampliação do acesso desta população às práticas corporais e de atividade física, através da implantação de programas estratégicos como a Academia da Saúde e o NASF, que tem em sua composição de educadores físicos, são inconclusivas no que tange às evidências científicas sobre o impacto destas estratégias na prática regular de atividade física e melhoria da qualidade de vida na população idosa.

\section{REFERÊNCIAS}

ABRAMS, M. Uma aferição difícil: a qualidade de vida. A saúde do Mundo, 4-11, 1974.

AGUIAR, J. B. DE; GURGEL, L. A. Investigação dos efeitos da hidroginástica sobre a qualidade de vida, a força de membros inferiores e a flexibilidade de idosas: um estudo no serviço social do comércio - Fortaleza. Rev. bras. educ. fís. Esp, 23(4), 335-344, 2009.

ALMEIDA, M. A. B.; GUTIERREZ, G. L.; MARQUES, R. Qualidade de vida: definição, conceitos e interfaces com outras áreas, de pesquisa - São Paulo: Escola de Artes, Ciências e Humanidades - EACH/USP, 2012.

AVERSAN, T. Influência da prática de atividade física na qualidade de vida do idoso: uma revisão bibliográfica. Revista Digital. Buenos Aires, 2012.

AUAD, M. A.; SIMÕES, R. P.; ROUHANI, S.; CASTELLO, V.; YOGI, L. S. Eficácia de um programa de exercícios físicos na qualidade de vida de mulheres com osteoporose. Arq. bras. ciênc. Saúde, 33(1),31-35, 2008.

BAPTISTA, V. C.; PALHARES, L. C.; OLIVEIRA, P. P. M.; FILHO, L. M. S.; VILARINHO, K. A. S.; SEVERINO, E. S. B. O. Teste de caminhada de seis minutos como ferramenta para avaliar a qualidade de vida em pacientes submetidos à cirurgia de revascularização miocárdica. Rev Bras Cir. Cardiovasc, 27(2), 231-239, 2012.

BENEDETTI, T. R. B.; GONÇALVES, L. H. T.; MOTA J. A. P. S. Uma proposta de Política Pública de Atividade Física para Idosos. Texto Contexto Enferm, 16(3), 387-98, 2007.

BORGES, M. R. D.; MOREIRA, A. K. Influências da prática de atividades físicas na terceira idade: estudo comparativo dos níveis de autonomia para o desempenho nas AVDs e AIVDs 
entre idosos ativos fisicamente e idosos sedentários. Motriz, rev. educação física, 15(3): 562$573,2009$.

BOUCHARD, C. Atividade física e obesidade. São Paulo: Manole, 2005.

BRASIL. Ministério da Justiça do Brasil. Estatuto do Idoso: Lei ${ }^{\circ} 10.741$, de $1^{\circ}$ de outubro de 2003. Brasília: Ministério da Justiça; 2003.

BRASIL. Ministério da Saúde. Política Nacional de Saúde da Pessoa Idosa. Portaria $\mathrm{n}^{\mathrm{o}}$ 2.528 de 19 de outubro de 2006. Ministério da Saúde, Imprensa Nacional, 2006.

BRASIL. Ministério da Saúde. Estatuto do Idoso. - 2. ed. rev. - Brasília: Editora do Ministério da Saúde, 2009.

BRASIL. Ministério da Saúde. VIGITEL Brasil, 2013. Disponível em: http://portal.saude.gov.br/portal/saude/profissional/area.cfm?idarea=1521. Acesso em: 28 mar. 2018.

BRENDEMEIER, S. M. L. Autonomia na velhice: concepções de idosos participantes de um programa de ação social. Estud. interdiscip. Envelhec, 16, 371-384, 2011.

CAMPOS, F. V. S.; PORTO, L. G. G. Qualidade de vida e nível de atividade física de pacientes em fase ambulatorial da reabilitação cardíaca. Rev. bras. ativ. fis. Saúde, 14(2), 2009.

CARVALHO, E. D.; VALADARES, A. L. R.; COSTA-PAIVA, L. H.; PEDRO, A. O.; MORAIS, S. S.; PINTO-NETO, A. M. Atividade física e qualidade de vida em mulheres com 60 anos ou mais: fatores associados. Rev. bras. ginecol. Obstet, 32(9): 433-440, 2010.

CARVALHO, J. A. M.; GARCIA, R. A. O envelhecimento da população brasileira: um enfoque demográfico, Caderno de Saúde Pública, 19(3),725-733, 2004.

CARLOS, K. P.; BELLI, B. S.; ALFREDO, P. P. Efeito do ultrassom pulsado e do ultrassom contínuo associado a exercícios em pacientes com osteoartrite de joelho: estudo piloto.

Fisioter. Pesquis. 19(3): 275-281, 2012.

COSTA, T.; SÍlVIA LOPES, S.; FERNÁNDEZ-LLIMÓS, F.; AMANTE, M. J.; LOPES, P. F. A Bibliometria e a Avaliação da Produção Científica: indicadores e ferramentas. In: Actas do Congresso Nacional de Bibliotecários, Arquivistas e Documentalistas. Disponível em: http://www.bad.pt/publicacoes/index.php/congressosbad/index. Acesso em: 20 jan. 2018.

DIAS, R. M. R.; GURJÃO, A. L. D.; MARUCCI, M. F. N. Benefícios do treinamento com pesos para aptidão física de idosos. Acta fisi trica, 13(2): 90-95, 2006.

ETCHEPARE, L. S.; PEREIRA, E. F., GRAUP, S.; YZINN, J. L. Terceira idade: aptidão física de praticantes de hidroginástica. Revista Digital, Buenos Aires, 65(9), 2004.

FERREIRA, C. C. Projeto de Prevenção de Quedas. In: Secretaria de Saúde de São Paulo, 2011. Disponível em: http://www.saude.sp.gov.br/resources/ipgg/resumo-de- 
projetos/projeto_prevencao_de_quegas_ipgg_-_nasf_indaia_kemel_e_atualpa.pdf . Acesso em: 28 mar. 2018.

FERREIRA, M. S.; MATSUDO, S.; MATSUDO, V.; BRAGGION, G. Efeitos de um programa de orientação de atividade física e nutricional sobre o nível de atividade física de mulheres fisicamente ativas de 50 a 72 anos de idade. Rev Bras Med Esporte, 11(3): 172$176,2005$.

FERREIRA, S. A; GUIMARÃES, M.; TAVEIRA, N. Reabilitação respiratória na DPOC: do treinamento de exercício para a vida real. J Bras Pneumol, 35(11): 1112-1115, 2009.

GONÇALVES, A. K. S.; CANÁRIO, A. C. G.; CABRAL, P. U. L.; SILVA, R. A. H.; SPYRIDES, M. H. C.; PAULO CÉSAR GIRALDO, P. C. Impacto da atividade física na qualidade de vida de mulheres de meia idade: estudo de base populacional. Rev. Bras. Ginecol. Obstet., 33(12), 2011.

GUIMARÃES J. M. N.; CALDAS C. P. A influência da atividade física nos quadros depressivos de pessoas idosas: uma revisão sistemática. Rev. Bras. Epidemiol, 481-492, 2006.

IMOTO, A. M.; PECCIN, M. S; TREVISANI, V. F. M. Exercícios de fortalecimento de quadríceps são efetivos na melhora da dor, função e qualidade de vida de pacientes com osteoartrite do joelho. Acta ortop. bras; 20(3): 174-179, 2012.

INSTITUTO BRASILEIRO DE GEOGRAFIA E ESTATÍSTICA - IBGE. Coordenação de população e indicadores sociais, Rio de Janeiro: IBGE, 2004.

INSTITUTO BRASILEIRO DE GEOGRAFIA E ESTATÍSTICA - IBGE. RIO DE JANEIRO, 2009. Departamento de População e Indicadores sociais. Disponível em: http://www.ibge.gov.br/home/presidencia/noticias/25072002pidoso.shtm. Acesso em: 23 jan. 2018.

KANNUS, P.; KHAN, K. M. Prevenção de queda e lesões subseqüentes em idosos: um longo caminho a percorrer em termos de pesquisa e prática. CMAJ. 5, 587-8, 2001.

KOKUBUN, E.; LUCIANO, E.; SIBUYA, C. Y.; QUEIROGA, M. R.; RIBEIRO, P. A. B.; SILVEIRA, R. F. Programa de atividade física em unidades básicas de saúde: relato de experiência no município de Rio Claro-SP. Rev. Bras. Ativ. Fís. Saúde, 12(1), 2007.

LIMA, F. F.; MIRANDA, R. C. V.; SILVA, R. C. R.; MONTEIRO, H. L.; YEN, L. S.; FAHUR, B. S.; PADULLA, S. A. T. Avaliação funcional pré e pós-programa de exercício físico de pacientes em hemodiálise. Medicina (Ribeirão Preto), 46(1), 2013.

LUSTOSA, L. P.; SILVA, J. P.; COELHO, F. M.; PEREIRA, D. S.; PARENTONI, A. N.; PEREIRA, L. S. M. Efeito de um programa de resistência muscular na capacidade funcional e na força muscular dos extensores do joelho em idosas pré-frágeis da comunidade: ensaio clínico aleatorizado do tipo crossover. Braz. j. phys. ther. (Impr.) ,15(4), 318-324, 2011.

MARAGONE, C. C. Benefícios da hidroginástica no envelhecimento. Escola Superior de Educação Física de Muzambinho, 2008. 
MENEZES, A. H T.; MIYAZATO, L.; ROSSLER, F.; ANTHERO, D. O. Oficina de Brinquedo: Uma Proposta de Intervenção para a Terceira Idade, 2007.

MOTA, J.; RIBEIRO, J. L.; CARVALHO, J.; MATOS, M. G. Atividade física e qualidade de vida associada à saúde em idosos participantes e não participantes em programas regulares de atividade física. Porto: Rev. bras. educ. fís. Esp, 20(3), 219-225, 2006.

MOZER, N. M. S.; OLIVEIRA, S. G.; PORTELA, M. R. Musicoterapia e exercícios terapêuticos na qualidade de vida de idosos institucionalizados. Estud. interdiscip. Envelhec, 16(2), 229-244, 2001.

NAVEGA, M. T.; OISHI, J. Comparação da qualidade de vida relacionada à saúde entre mulheres na pós-menopausa praticantes de atividade física com e sem osteoporose. Rev. Bras. Reumatol, 47(4), 258-264, 2007.

OLIVEIRA, A. C.; OLIVEIRA, N. M. D.; ARANTES, P. M. M.; ALENCAR, M. A. Qualidade de vida em idosos que praticam atividade física: uma revisão sistemática. Rev. bras. geriatr. Gerontol, 13(2), 301-312, 2010.

PEDRINELLI, A.; GARCEZ-LEME, L. E.; NOBRE, R. S. A. O efeito da atividade física no aparelho locomotor do idoso. Rev. bras. ortop, 44(2): 96-101, 2009.

PIMENTA, F. A. P.; SIMIL, F. F.; TÔRRES, H. O. G.; AMARAL, C. F. S.; REZENDE, C. F.; COELHO, T. O. Avaliação da qualidade de vida de aposentados com a utilização do questionário SF-36. Rev. Assoc. Med. Bras., fev., 54(1),55-60, 2008.

PITANGA, F. J. G. Epidemiologia, atividade física e saúde. Rev. Bras. Ciên. e Mov. 10(3), 2005.

PRADO, R. A. A influência dos exercícios resistidos no equilíbrio, mobilidade funcional e na qualidade de vida de idosas. Mundo saúde (Impr.), 34(2), 183-191, 2010.

PUCCI, G. C. M. F.; RECHI, C. R.; FERMINO, R. C.; REIS, R. S. Associação entre atividade física e qualidade de vida em adultos. Rev. Saúde Pública, 46(1), 166-179, 2012.

RITTER, A. L.; AZEVEDO, D. A função da instrução técnica na qualidade de movimento de idosas obesas. Salusvita, 26(1), 77-88, 2007.

SANTOS, S. R.; SANTOS, I. B. C.; FERNANDES, M. G. M.; HENRIQUE, S. M.Qualidade de vida dos idososna comunidade: aplicação da Escala de Flanagan. Rev. Latino Am. Enfermagem, 10 (6),757-64, 2004.

SANTOS J. L. F.; LEBRÃO, M. L.; DUARTE, Y. A. O.; LIMA, F. D. Functional performance of the elderly in instrumental activities of daily living: an analysis in the municipality of São Paulo, Cad. Saúde Pública, 24(4), 879-886, 2008.

SEBASTIÃO, E.; CHRISTOFOLETTI, G.; GOBBI, S.; HAMANAKA, A. Y. Y. Atividade física e doenças crônicas em idosos de Rio Claro - SP. Motriz, 14(4), 381 - 388, 2008. 
SECRETARIA ESTADUAL DE SAÚDE. Núcleo de Informações em Saúde- NIS. Sistema de informação sobre mortalidade no Rio Grande do Sul. Disponível em:

http://www.saude.rs.gov.br/wsa/portal/index.jsp?menu=organograma\&cod=746. Acesso em: 22 fev. 2018.

SILVA, H. O.; CARVALHO, M, J. A. D.; LIMA, F. E. L.; RODRIGUES, L. V. Perfil epidemiológico de idosos frequentadores de grupos de convivência no município de Iguatu, Ceará. Rev. bras. Geriatr. Gerontol, 14(1), 123-133, 2011.

SPIRDUSO, W. W. Dimensões físicas do envelhecimento. 1 ed. Barueri: Manole, 2005. 482 p.

SUDBRACK, A. C.; SARMENTIO L. R. Efetividade do exercício na claudicação. Rev. bras. cardiol. Invasiva, 15(3), 261-266, 2007.

TAIROVA, O. S.; LORENZI, D. R. S. A influência do exercício físico na qualidade de vida de mulheres na pós-menopausa: um estudo caso-controle. Caxias do Sul, Rev. bras. geriatr. Gerontol, 14(1), 135-145, 2011.

TERENCE, A. C. F.; FILHO, E. E. Abordagem quantitativa, qualitativa e a utilização da pesquisa-ação nos estudos organizacionais. XXVI ENEGEP - Fortaleza, CE, Brasil, 9 a 11 de outubro de 2006.

TOSCANO, J. J. O.; OLIVEIRA, A C. C. Qualidade de vida em idosos com distintos níveis de atividade física. Revista brasileira de medicina do esporte, 15(.3), 169-173, 2009.

VANTI, N. Da bibliometria à webometria: uma exploração conceitual dos mecanismos utilizados para medir o registro da informação e a difusão do conhecimento. Ci Inf., 152-62, 2002.

ZANCHET, R. C.; VIEGAS, C. A. A.; LIMA, T. A. Eficácia da reabilitação pulmonar na capacidade de exercício, força da musculatura inspiratória e qualidade de vida de portadores de doença pulmonar obstrutiva crônica. J Bras Pneumol, 31(2), 118-124, 2005.

\section{Como referenciar este artigo}

ARAÚJO, D. P.; SOARES, S. L.; PALÁCIO, D. Q. A. A relação da atividade física e a qualidade de vida na população idosa: análise da produção cientifica brasileira. Temas em Educ. e Saúde, Araraquara, v. 14, n. 2, p. 242-253, jul./dez., 2018. e-ISSN 2526-3471. DOI: 10.26673/tes.v14i2.11520

Submetido em: 03/07/2018

Revisões requeridas: 18/08/2018

Aprovado em: 10/10/2018 